\title{
瀻 \\ SISTEMA REPRODUCTIVO Y BIOLOGÍA FLORAL DE LANTANA CAMARA (VERBENACEAE) EN UNA POBLACIÓN RIBEREÑA DEL RÍO dE LA PLATA
}

\author{
REPRODUCTIVE SYSTEM AND FLORAL BIOLOGY OF LANTANA CAMARA \\ (Verbenaceae) in a Riparian population of the Río de la Plata \\ María T. Amela García ${ }^{1,2}\left(\mathbb{D}\right.$, Diego Aquino ${ }^{1}$, Agustina Bouza1(D, \\ Mariana C. Grohar ${ }^{* 1}$ D, Anahí N. Herrera Cano ${ }^{1} \mathbb{D}$, Maiten A. Lafuente Diaz ${ }^{1} \mathbb{D}$, \\ María B. Miguez ${ }^{1}$, Victoria Scorza ${ }^{1}$ (D) y Marina Chamer ${ }^{1,3}$ (D)
}

\begin{abstract}
1. Universidad de Buenos Aires, Facultad de Ciencias Exactas y Naturales, Departamento de Biodiversidad y Biología Experimental, Grupo de Biología Reproductiva en Plantas Vasculares. Buenos Aires, Argentina.

2. INMIBO (UBA-CONICET). Buenos Aires, Argentina.

3. Universidad de Buenos Aires, Facultad de Agronomía, Departamento de Recursos Naturales y Ambiente, Buenos Aires, Argentina.
\end{abstract}

*mariana.grohar@gmail.com

\section{Citar este artículo}

AMELA GARCÍA, M. T., D. AQUINO, A. BOUZA, M. C. GROHAR, A. N. HERRERA CANO, M. LAFUENTE DIAZ, M. B. MIGUEZ, V. SCORZA \& M. CHAMER. 2019. Sistema reproductivo y biología floral de Lantana camara (Verbenaceae) en una población ribereña del Río de la Plata. Bol. Soc. Argent. Bot. 54: 29-42.

DOI: http://dx.doi. org/10.31055/1851.2372.v54. n1.23578

Recibido: 17 Octubre 2018

Aceptado: 7 Enero 2019

Publicado: 28 Marzo 2019

Editora: Ana Calviño (D)

ISSN versión impresa 0373-580X ISSN versión on-line 1851-2372

\section{SUMMARY}

Background and aims: The floral biology of Lantana camara, a native species of Central and South America and invasive cosmopolitan, still presents poorly-known and controversial aspects. The aim of this work was to determine the reproductive system, floral attributes and pollinators in a coastal population of Río de la Plata.

M\&M: The usual methods in pollination biology were employed. The study was performed in Ciudad Universitaria, Ciudad Autónoma de Buenos Aires, Argentina, during the spring of 2012.

Results: The flowers emit mild and sweet odor by osmophores arranged around the floral tube entrance, in coincidence with the absorption-reflection pattern in the UV spectrum. Nectar accumulates in the floral tube, probably secreted by trichomes situated at the petals base. During anthesis, three floral phases succeed, distinguished by changes in corolla coloration, aroma intensity, reward quantity and anther and stigma maturity. The psychophilous flowers were visited by diurnal lepidopterans and by hymenopterans, dipterans and hummingbirds. The highest reproductive success occurred with free pollination; the self-incompatibility and $\mathrm{P} / \mathrm{O}$ indexes showed partial self-compatibility and facultative xenogamy, respectively.

Conclusions: $L$. camara is capable of successful spontaneous self-pollination, probably one reason of its global spread. Due to their activity on the flowers, visited floral phase, pollen load and frequency of visits, only the butterflies Agraulis vanillae maculosa, Dryas iullia alcionea and Cymaenes sp. would pollinate in this site.

\section{KEY WORDS}

Lantana camara, facultative xenogamy, Lepidoptera, reproductive success, floral phases, UV reflectance pattern, osmophores.

\section{RESUMEN}

Introducción y objetivos: La biología floral de Lantana camara L., nativa del Centro y Sur de América e invasora cosmopolita, tiene aspectos desconocidos y controvertidos. El objetivo de este trabajo fue determinar el sistema reproductivo, atributos florales y polinizadores en una población ribereña del Río de la Plata.

M\&M: Se emplearon los métodos usuales de la biología de la polinización. El estudio se realizó en Ciudad Universitaria, Ciudad Autónoma de Buenos Aires, Argentina, en la primavera de 2012 .

Resultados: Las flores emiten olor leve y dulce mediante osmóforos que rodean la entrada del tubo floral, coincidiendo con el patrón de absorción-reflexión en el espectro UV. El néctar se acumula en el tubo corolino, secretado probablemente por tricomas situados en la base. Durante la antesis, se suceden tres fases florales, distinguibles por cambios en la coloración de la corola, intensidad del aroma, cantidad de recompensa y madurez de las anteras y el estigma. Las flores, psicófilas, recibieron visitas de lepidópteros diurnos, y también de himenópteros, dípteros y colibríes. El mayor éxito reproductivo ocurrió con polinización libre; los índices de autocompatibilidad y $\mathrm{P} / \mathrm{O}$ indicaron autocompatibilidad parcial y xenogamia facultativa, respectivamente.

Conclusiones: L. camara se autopoliniza espontáneamente, probablemente una causa de su propagación global. Debido a su actividad en las flores, fase floral visitada, carga polínica y frecuencia de visitas sólo las mariposas Agraulis vanillae maculosa, Dryas iullia alcionea y Cymaenes sp. polinizarían en este sitio.

\section{Palabras clave}

Lantana camara, xenogamia facultativa, Lepidoptera, éxito reproductivo, fases florales, patrón de reflexión UV, osmóforos. 


\section{INTRODUCCIÓN}

Las verbenáceas poseen una distribución principalmente tropical y subtropical, ocurriendo unos escasos géneros en regiones templadas (Heywood, 1985). El género Lantana L. incluye 150 especies tropicales en América y África, de las cuales 19 habitan la Argentina (De Marzi, 2006). Lantana camara L. es nativa de Sur y Centro América, habiéndose naturalizado en las islas del Pacífico, Australia, Nueva Zelanda, África y Asia meridional (Morton, 1994; Baars \& Neser, 1999; Thorp \& Lynch, 2000). En la Argentina, se distribuye en las provincias de Buenos Aires, Córdoba, Corrientes, Entre Ríos, Formosa, Jujuy, Misiones, Salta, Santiago del Estero, Santa Fe y Tucumán (Anton \& Zuloaga, 2012). Este taxón es considerado una de las 100 especies exóticas invasoras más dañinas del mundo según la Comisión de Supervivencia de Especies (CSE) de la Unión Mundial para la Naturaleza (UICN) (Lowe et al., 2000), y es reconocida como una amenaza a los ecosistemas de Australia (Haseler \& Kassulke, 1996), las islas Solomon y Vanuatu (Harley, 1974), entre otros. La invasión de la especie se ve favorecida por el disturbio de los hábitats naturales a través del incremento de la explotación forestal (Wells \& Stirton, 1988) y la introducción de vertebrados exóticos (Thaman, 1974; Denton et al., 1991; Fensham et al., 1994). A pesar de los esfuerzos de manejo, continúa siendo un problema (Bhagwat et al., 2012) en muchas regiones del planeta (Goncalves et al., 2014), en particular porque en forma drástica provoca cambios en la estructura de la comunidad (Sundaram \& Hiremath, 2012), reduciendo la cobertura de forraje, la producción de cultivos y la disponibilidad de recursos nativos (Shackleton et al., 2017). En nuestro país, se ha estudiado la dispersión de sus semillas considerando su carácter invasor (Grilli \& Galletto, 2009).

Por otra parte, L. camara es tóxica para los vertebrados y puede causar fotosensibilidad en ganado vacuno u ovino (Tokarinia et al., 1999; Matienzo et al., 2003; Brito et al., 2004). Sin embargo, es utilizada en la medicina tradicional en Latinoamérica (Hernández et al., 2003; Rivera Amita et al., 2004; Carrere, 2006; Hernández et al, 2010). Posee terpenoides, esteroides y alcaloides (Ghisalberti, 2000) que le otorgan a la hoja propiedades antimicrobianas, fungicidas $\mathrm{y}$ nematicidas (Chavan \& Nikam, 1982; Sharma \& Sharma, 1989; Begum et al., 2000). Además, es empleada como ornamental para cercos vivos (debido a su fácil mantenimiento e inflorescencias vistosas), como cobertor de suelos en jardinería y como leña (Singh et al., 1984; Ramírez-Hernández et al., 2012).

Lantana camara es un arbusto con flores dispuestas en cimas umbeliformes, pentámeras, con los sépalos soldados y los pétalos formando una corola hipocraterimorfa, levemente zigomorfa, cuyo limbo presenta dos labios esbozados de dos y tres lóbulos, respectivamente, cuatro estambres didínamos unidos al tubo corolino, anteras de dehiscencia longitudinal, gineceo súpero y dímero. El fruto es una drupa globular púrpura oscuro o negra, con semillas de 1,5 mm de longitud (Holm et al., 1977; Troncoso, 1979; Sastri \& Kavathekar, 1990).

Respecto de su biología floral, se sabe que sus flores son delicadas, de colores vívidos y erectas; la corola forma un margen plano, angosto, poco disecto y un tubo angosto en el que se oculta el néctar, con guías de néctar simples, olor débil, fresco y agradable, anteras fijas y antesis diurna sin cierre nocturno (Holm et al., 1977). Durante la antesis, el estigma secreta una sustancia mucilaginosa y el color de la corola vira del amarillo al rojo (Mathur \& Mohan Ram, 1978; Valla, 1979; Weiss, 1995; Caroprese Araque et al., 2011), pasando por el naranja (De Marzi, 2006). Cada flor constituye una unidad del tipo trompeta y el típico patrón polinizado por lepidópteros diurnos (psicofilia) (Faegri \& Van Der Pijl, 1979). Ha sido mencionada como especie blanco de mariposas en ambientes urbanos de Buenos Aires (Núñez Bustos, 2008). La especificidad de éstas fue analizada por Schemske (1976) en base a la comparación entre algunas dimensiones de los insectos y de las flores, si bien no corroboraron el contacto con anteras ni estigmas así como tampoco el transporte de polen. Las mariposas son vectores poco eficaces en la remoción y depósito de polen debido a los escasos granos que transportan y a que algunas exhiben poca constancia floral (Percival, 1965), incluso hurtan néctar (Wiklund et al., 1979); sin embargo, comparadas con las abejas, tienden a volar distancias mayores entre visitas consecutivas $\mathrm{y}$, por tanto, a transferir polen xenógamo (Herrera, 1987). A pesar de las flores psicófilas de L. camara, 


\section{T. Amela García et al. - Biología reproductiva de Lantana camara}

distintos autores han aseverado que Apis mellifera la poliniza en forma eficiente en Australia, donde ambas especies son exóticas (Goulson \& Derwent, 2004, entre otros). Por el contrario, Mathur \& Mohan Ram (1978) afirmaron que en India las mariposas son polinizadores eventuales y que, en cambio, los trips son regulares, aunque no corroboraron la transferencia de polen al estigma por estos últimos.

En cuanto a su sistema de autoincompatibilidad no existe consenso. Según Barrows (1976), bajo condiciones controladas, L. camara sólo produce frutos por polinización cruzada; según Mathur \& Mohan Ram (1978), es autocompatible, aunque necesita ser polinizada por vectores. Neal (1999) señaló que en la especie existe autopolinización. Granja Barros et al. (2001) comentaron que algunas de las variedades ornamentales son autoincompatibles.

Ante la falta de claridad y el desconocimiento sobre algunos aspectos de la biología reproductiva de $L$. camara y debido a la importancia de generar este conocimiento, ya sea para poder aplicarlo contra su poder invasivo o para emplearlo para cultivarla por sus utilidades, el objetivo del presente trabajo fue la descripción detallada de las características florales, la determinación del sistema reproductivo y el reconocimiento de los polinizadores en una población de la ribera del Río de la Plata.

\section{Materiales y MÉtodos}

Área de Estudio. El estudio se llevó a cabo en una población natural en el Campo Experimental de la Facultad de Ciencias Exactas y Naturales de la Universidad de Buenos Aires (FCEyN-UBA), en la Ciudad Autónoma de Buenos Aires (Argentina) desde el 17 hasta el 26 de octubre de 2012.

Sistema Reproductivo. Para determinarlo, se realizó un experimento de polinización controlada, aplicando los siguientes tratamientos: autogamia espontánea (inflorescencia embolsada), autopolinización inducida (estigmas polinizados manualmente con polen autógamo), xenogamia inducida (estigmas polinizados con polen alógamo) y polinización abierta (control). Para cada tratamiento se utilizaron 7 inflorescencias con capullos próximos a la antesis, las cuales se embolsaron con bolsas de organza, a excepción del tratamiento de polinización abierta, en que solamente se rotularon con una etiqueta colgante del pedúnculo. Al mes, se contabilizaron los frutos y las semillas obtenidos en cada tratamiento y se calcularon los porcentajes de fructificación y llenado de frutos. Con los datos obtenidos se calcularon los índices de autoincompatibilidad ISI (Ruiz Zapata \& Arroyo, 1978) y éxito reproductivo relativo (ERR), calculado como cantidad de flores/ cantidad de frutos $\mathrm{x}$ cantidad de óvulos/cantidad de semillas para cada tratamiento (Dafni, 1992). Además, se calculó el estimador P/O (Cruden, 1977), para lo cual se fijaron capullos próximos a la antesis y se contaron los óvulos y los granos de polen, éstos últimos con cámara de Newbauer.

Caracteres florales. Para determinar las fases florales, se observaron los cambios en 20 inflorescencias de cinco plantas distribuidas en tres parches durante cinco días. Se analizó el estado de maduración de las anteras, la receptividad estigmática y los atractivos florales (color, olor, néctar y polen). El estado de maduración de las anteras se determinó según el grado de dehiscencia $\mathrm{y}$, una vez abierta, se registró la presencia/ abundancia de polen en las mismas. La receptividad estigmática se evaluó según el método de Osborn et al. (1988), para lo que se colocó una gota de peróxido de hidrógeno sobre el estigma libre de polen, considerando positiva la reacción al observar burbujeo. Se comparó la coloración de la corola con la carta de colores de Kelly (1965). Además, se registró el patrón de reflexión UV mediante el uso de un filtro Kodak 18 A con una cámara fotográfica réflex manual Pentax K-1000.

Para detectar las piezas productoras de aroma, a dos flores de cada color se les separaron las piezas, las cuales se colocaron en frascos de vidrio, que se cerraron herméticamente. Tras 1 hora, se realizó una prueba olfatoria con siete personas. La intensidad se valoró en una escala de 1 a 10 , siendo el valor 1 el menos intenso, y se calculó la moda estadística. Además, se tiñeron 3 flores de cada estadio con rojo neutro para la localización de osmóforos, y con safranina para la detección de estructuras secretoras.

Para analizar el patrón de secreción del néctar, se embolsaron tres grupos de capullos con bolsas de organza y, cuando abrieron, se midió el volumen de néctar cuando alcanzó cada grupo cada una de las fases. Para ello se extrajo el néctar con 
capilares de 0,5 mm de diámetro, se midió la altura alcanzada por el néctar en el capilar y se calculó el volumen con la fórmula del cilindro. Además, se registró la concentración de azúcares totales con un refractómetro de mano.

Se determinó la predominancia de reservas del polen fresco sometiendo los granos por 5 minutos a 3 tratamientos: agua destilada (control), Sudán IV (detección de lípidos) y Lugol (detección de almidón), observándose con microscopio óptico la reacción respecto del control. Una vez conocida la reserva principal, se determinó la viabilidad de los granos de polen por el método de reservas principales (Amela García, 1999) en flores al inicio y al final de la antesis: se tiñeron granos de polen con una gota de Lugol y, luego de unos minutos, se observó al microscopio óptico. Se contabilizó la cantidad de granos viables y se calculó el porcentaje.

Visitantes florales. Se registró la visita de animales a las flores (identidad, frecuencia, duración y comportamiento) cada hora, de 8 a 19 horas, durante los días 17, 18, 24 y 25 de octubre en 10 plantas. Se capturaron ejemplares representativos para la determinación taxonómica y el análisis de la carga polínica; esta última se procesó mediante microacetólisis (Genise et al., 1990), observándose con microscopio óptico y con el microscopio electrónico de barrido Zeiss Supra 40 FESEM del Centro de Microscopías Avanzadas (FCEyN, UBA). Para poder identificar los granos de polen transportados por los visitantes, se recolectaron ejemplares de herbario de las especies con posible polinización biótica alrededor de los parches principales de L. camara para la confección de preparados de polen de referencia mediante acetólisis (Erdtman, 1952). La función de cada visitante se categorizó en base al transporte de polen y a su actividad en las flores acorde con Inouye (1980): hurtador, ladrón, polinizador. Una vez reconocidos los polinizadores, la importancia relativa de cada uno se consideró en base a su frecuencia.

Condiciones meteorológicas. Se registraron a través de los informes diarios de la Estación Meteorológica Automática Ciudad Universitaria (DCAO, FCEyN, UBA), ubicada en el mencionado campo experimental, con datos actualizados cada 10 minutos.

Análisis estadísticos. A fin de evaluar las diferencias entre medias, para la viabilidad polínica se realizó un contraste de pares empleando la prueba $t$ de student; para la producción de semillas entre los distintos tratamientos y para la frecuencia de visitas a las distintas fases florales se usó la prueba de Kruskal-Wallis y el test de Wilcox para contrastes de a pares (R Development Core Team, 2009).

\section{Resultados}

Sistema reproductivo. Se obtuvieron frutos con semillas en todos los tratamientos realizados. Si bien la mayor cantidad se produjo por polinización libre, reflejándose en un mayor índice ERR (Tabla 1), las diferencias no fueron significativas para los frutos $(\mathrm{K}-\mathrm{W}=6,26 ; P=0,09)$, ni para las semillas $(\mathrm{K}-\mathrm{W}=5,13 ; P=0,16)$. El índice ISI fue $0,57 \mathrm{y}$ el índice $\mathrm{P} / \mathrm{O} 786 \pm 426(\mathrm{~N}=7)$.

Tabla 1. Productividad por inflorescencia de L. camara según los distintos tratamientos de polinización. Media porcentual y desvío estándar de la producción de frutos y semillas.

\begin{tabular}{|cccccc|}
\hline Tratamiento & $\begin{array}{c}\text { Autogamia } \\
\text { espontánea }\end{array}$ & $\begin{array}{c}\text { Autogamia } \\
\text { inducida }\end{array}$ & $\begin{array}{c}\text { Xenogamia } \\
\text { inducida }\end{array}$ & $\begin{array}{c}\text { Polinización } \\
\text { libre }\end{array}$ \\
\hline$N^{\circ}$ de inflorescencias & 6 & 6 & 5 & 4 \\
$N^{\circ}$ de óvulos & 246 & 244 & 206 & 170 \\
$N^{\circ}$ de flores & 123 & 122 & $13 \pm 15$ & $25 \pm 18$ \\
\% de frutos & $4 \pm 8$ & $6 \pm 8$ & $13 \pm 1$ & $22 \pm 1$ \\
\hline ERR de semillas & $10 \pm 1$ & $16 \pm 1$ & 0,0086 & 0,0320 \\
\hline
\end{tabular}




\section{T. Amela García et al. - Biología reproductiva de Lantana camara}

Antesis, fases florales y atractivos. En los capullos próximos a la antesis, las anteras estaban dehiscentes y el estigma receptivo, éste por debajo del nivel de las anteras. Una vez abierta la flor, se sucedieron 3 fases florales: temprana, intermedia y tardía, con características distintivas de color (Fig. 1), maduración de anteras, receptividad estigmática y producción de néctar (Tabla 2). La concentración del néctar fue $22 \% \pm 2(N=2)$ y se halló en las dos primeras fases, más abundante en la primera (Tabla 2). Abrieron $3 \pm 1(N=20)$ capullos por inflorescencia por día. La apertura de capullos ocurrió principalmente por la mañana hasta el mediodía (Fig. 2) y las flores permanecieron abiertas hasta el día siguiente, sin cerrar durante la noche; el promedio de flores abiertas por día fue 7 $\pm 0(N=20)$. La cantidad de flores en cada fase fue bastante variable entre inflorescencias en un mismo día en todo momento, evidenciado por los desvíos (Fig. 2). El pasaje de una fase a otra fue más lento el día 18, hallándose flores en fase 2 recién a las 11 hs y sin alcanzar la fase 3 dentro de las horas de luz de ese día, a diferencia de los días 24 y 25 , en que el cambio de fases fue más homogéneo, hallándose flores en todas las fases desde las 8 hs (Fig. 2). La maduración de la inflorescencia es centrípeta (Fig. 1). Según la fase en la que se hallaban la mayoría de las flores de una inflorescencia en un determinado momento, también se aplicaron los términos temprana, intermedia y tardía a las inflorescencias.

Se observó un leve contraste entre la entrada del tubo floral y el resto de la corola, tanto en el espectro visible como UV (Fig. 1E-F). El olor, dulce y leve, fue emitido en todas las fases, más intensamente en la temprana (Fig. 3A); en particular, cáliz y corola emitieron con más intensidad (Fig. 3B). Se detectaron osmóforos en el limbo alrededor de la entrada del tubo floral, siendo la intensidad de la tinción mayor en la fase
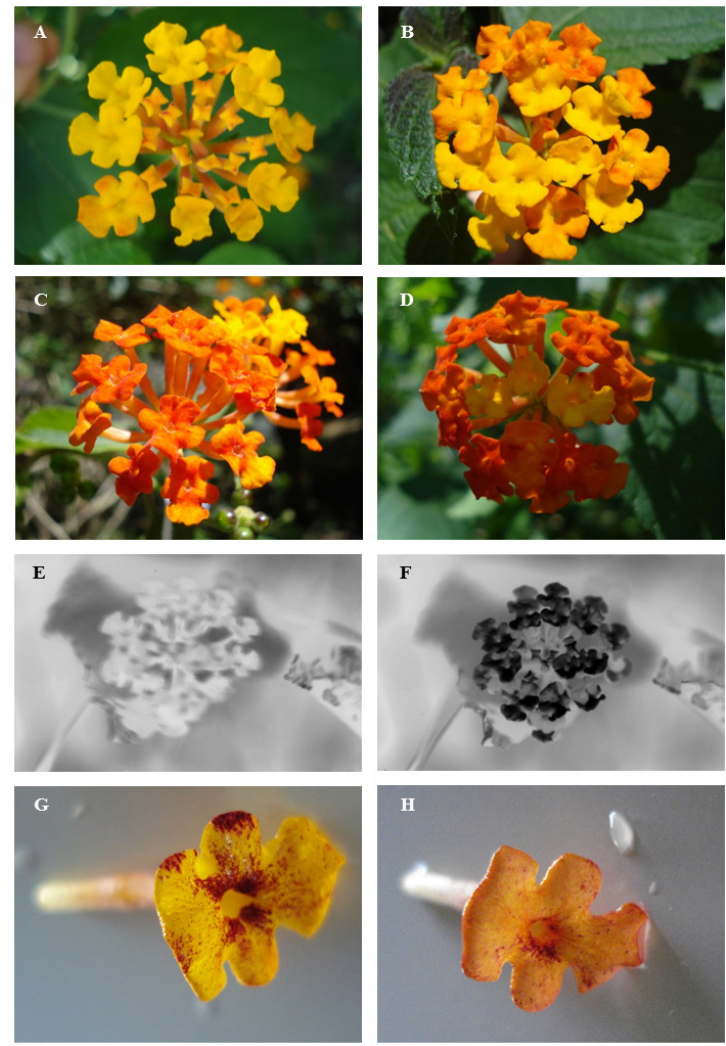

Fig. 1. Atractivos visuales y olfativos de las flores de L. camara. A-D: Coloración en el espectro visible. A: Inflorescencia en fase temprana. B y C: Fase intermedia. D: Fase tardía. E-F: Patrón de reflexión y absorción en el espectro visible (E) y en el UV (F). G: Osmóforos en una flor en fase temprana. H: Osmóforos en una flor en fase intermedia.

temprana (Fig. 1G-H). Se encontraron numerosos tricomas no capitados en el interior de la base del tubo corolino que se colorearon con safranina. La principal sustancia de reserva en los granos de

Tabla 2. Fases florales de $L$. camara. Se indica color de la corola, volumen de néctar por flor (media \pm DS, $N=2$ ), dehiscencia de anteras y receptividad estigmática.

\begin{tabular}{|ccccc|}
\hline Fase & Color & Néctar $(\boldsymbol{\mu l})$ & Anteras & Estigma \\
\hline Temprana & amarillo anaranjado vívido & $4,5 \pm 0,1$ & Dehiscentes & Receptivo \\
Intermedia & naranja vívido & $3,7 \pm 0,1$ & Dehiscentes & No receptivo \\
Tardía & rojo anaranjado profundo & 0 & Marchitas & No receptivo \\
\hline
\end{tabular}



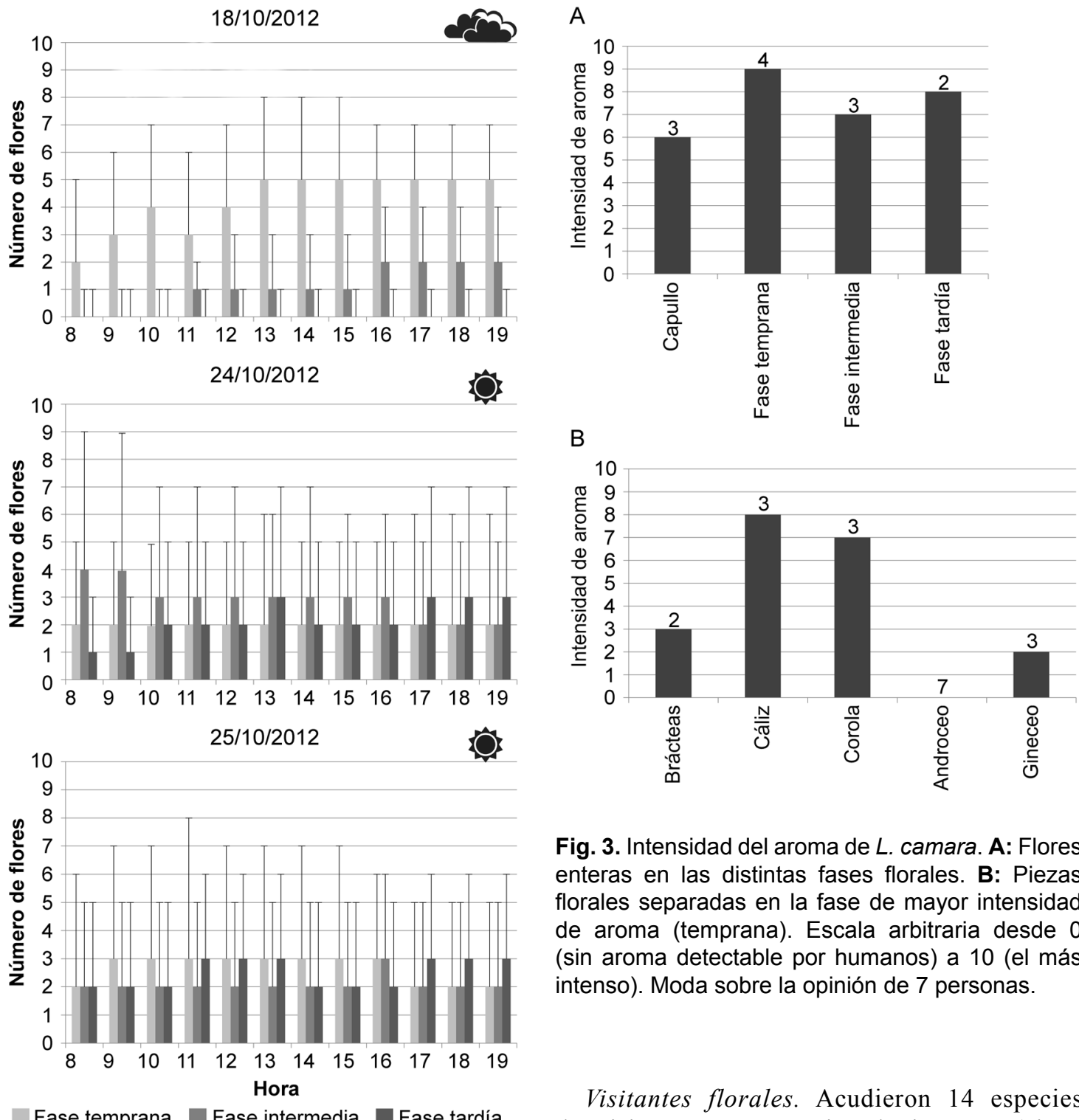

Fig. 3. Intensidad del aroma de $L$. camara. A: Flores enteras en las distintas fases florales. B: Piezas florales separadas en la fase de mayor intensidad de aroma (temprana). Escala arbitraria desde 0 (sin aroma detectable por humanos) a 10 (el más intenso). Moda sobre la opinión de 7 personas.

Fig. 2. Sucesión de las fases florales de L. camara. Promedio de flores en cada fase a lo largo de la antesis en días con distintos niveles de irradiación solar. Por cuestiones de claridad, sólo se graficaron los desvíos positivos.

polen fue almidón. Si bien la viabilidad polínica fue mayor en la fase temprana comparada con la tardía ( $47 \pm 17$ vs. $43 \pm 11)$, las diferencias en los porcentajes no fueron significativas $(P=1 ; \alpha=$ 0,$05 ; N=3,34$ granos).

Visitantes florales. Acudieron 14 especies de visitantes: 13 especies de insectos (cinco pertenecientes a Lepidoptera, siete a Hymenoptera, una a Diptera) y un ave (Apodiformes) (Tabla 3). Los días 17 y 18 la temperatura fue más elevada, la humedad fue más baja y la irradiación fue mayor que los días 24 y 25 (Tabla 3). Entre los visitantes observados, predominaron las visitas de lepidópteros, destacándose Agraulis vanillae maculosa y Dryas iulia alcionea, con más frecuencia los días soleados (Tabla 3). Las flores en las distintas fases florales recibieron significativamente diferente cantidad de visitas $(\mathrm{K}-\mathrm{W}=22,52 ; P<0.0001)$ : las flores más visitadas fueron las que se hallaban en fase temprana, seguidas por la intermedia y la tardía (Tabla 4). 
Tabla 3. Condiciones ambientales, fases florales y visitas en los días de muestreo.

\begin{tabular}{|c|c|c|c|c|c|c|}
\hline \multicolumn{2}{|c|}{ Fechas } & $17 / 10$ & $18 / 10$ & $24 / 10$ & $25 / 10$ & Total \\
\hline \multicolumn{7}{|c|}{ Condiciones meteorológicas } \\
\hline & Temperatura promedio $\left({ }^{\circ} \mathrm{C}\right)$ & 17,3 & 16,8 & 18,5 & 20,1 & - \\
\hline & Temperatura máxima $\left({ }^{\circ} \mathrm{C}\right)$ & 21,3 & 20,5 & 24,4 & 25,9 & - \\
\hline & Temperatura mínima $\left({ }^{\circ} \mathrm{C}\right)$ & 14,7 & 11,1 & 12,1 & 13,1 & - \\
\hline & Humedad relativa $(\%)$ & 68,3 & 76,8 & 51,7 & 60,9 & - \\
\hline & Irradiancia & Nublado & Nublado & Soleado & Soleado & - \\
\hline \multicolumn{7}{|c|}{ Cantidad de flores visitadas } \\
\hline & Fase temprana & 4 & 20 & 171 & 89 & 284 \\
\hline & Fase intermedia & 1 & 17 & 13 & 5 & 36 \\
\hline & Fase tardía & 1 & 4 & 1 & 0 & 6 \\
\hline \multicolumn{2}{|c|}{ Total de flores visitadas } & 6 & 41 & 185 & 94 & 326 \\
\hline \multicolumn{2}{|c|}{ Especies visitantes } & \multicolumn{5}{|c|}{ Número de visitas } \\
\hline \multirow{17}{*}{ 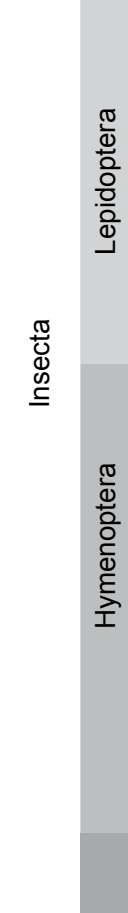 } & Agraulis vanillae maculosa & 33 & 9 & 1 & 14 & 57 \\
\hline & Dryas iulia alcionea & 33 & 37 & 42 & 25 & 137 \\
\hline & Ortilia ithra & 17 & 0 & 12 & 9 & 38 \\
\hline & Cymaenes sp. & 0 & 0 & 11 & 16 & 27 \\
\hline & Epargyreus tmolis & 0 & 26 & 2 & 0 & 28 \\
\hline & Cantidad de visitas de Lepidoptera & 83 & 72 & 68 & 64 & 287 \\
\hline & Subtotal de especies de Lepidoptera & 3 & 3 & 5 & 4 & 5 \\
\hline & Apis mellifera & 0 & 0 & 2 & 6 & 8 \\
\hline & Aff. Halictidae & 0 & 0 & 0 & 3 & 3 \\
\hline & Aff. Andrenidae & 0 & 0 & 0 & 2 & 2 \\
\hline & Bombus sp. & 0 & 5 & 6 & 5 & 16 \\
\hline & Xylocopa sp. & 0 & 0 & 2 & 0 & 2 \\
\hline & Polystes sp. & 0 & 14 & 3 & 1 & 18 \\
\hline & Brachygastra lecheguana & 0 & 5 & 1 & 1 & 7 \\
\hline & Cantidad de visitas de Hymenoptera & 0 & 24 & 14 & 18 & 56 \\
\hline & Subtotal de especies de Hymenoptera & 0 & 3 & 5 & 6 & 7 \\
\hline & $\begin{array}{l}\text { Cantidad de visitas de Diptera } \\
\text { (Syrphidae) }\end{array}$ & 17 & 2 & 5 & 0 & 24 \\
\hline Aves & $\begin{array}{l}\text { Cantidad de visitas de Aves } \\
\text { (Apodiformes, Chlorostilbon lucidus) }\end{array}$ & 0 & 2 & 13 & 18 & 33 \\
\hline
\end{tabular}

Se encontró polen de $L$. camara sólo en tres de las cuatro especies de lepidópteros que transportaban polen (Fig. 4), particularmente en la cabeza, en baja cantidad y junto con granos de otras especies. En los ápidos y véspidos se hallaron granos de polen de otras especies de plantas, en las patas y el abdomen (Fig. 4).

La variación principal en las condiciones meteorológicas fue en la irradiación. La cantidad 
Tabla 4. Cantidad de visitas de los distintos taxa a flores de $L$. camara según las fases florales. Letras distintas indican diferencias significativas $(P=0,05)$.

\begin{tabular}{|c|c|c|c|}
\hline Especies visitantes & Fase temprana & Fase intermedia & Fase tardía \\
\hline Agraulis vanillae maculosa & 69 & 6 & 0 \\
\hline Dryas iulia alcionea & 83 & 14 & 0 \\
\hline Ortilia ithra & 24 & 1 & 0 \\
\hline Cymaenes sp. & 51 & 3 & 0 \\
\hline Epargyreus tmolis & 15 & 2 & 0 \\
\hline Apis mellifera & 10 & 2 & 0 \\
\hline Aff. Halictidae & 5 & 0 & 1 \\
\hline Aff. Andrenidae & 5 & 0 & 0 \\
\hline Bombus sp. & 17 & 0 & 1 \\
\hline Xylocopa sp. & 0 & 0 & 0 \\
\hline Polystes sp. & 3 & 4 & 2 \\
\hline Brachygastra lecheguana & 6 & 6 & 1 \\
\hline Syrphidae & 6 & 2 & 1 \\
\hline Chlorostilbon lucidus & 51 & 1 & 0 \\
\hline Total & $345^{b}$ & $41^{\mathrm{b}}$ & $6^{c}$ \\
\hline
\end{tabular}

de especies visitantes fue mayor los días soleados, así como la cantidad de visitas de las abejas; en cambio, la cantidad de visitas de los lepidópteros en días soleados fue levemente menor (Tabla 3).

La actividad de los visitantes florales en las flores se describe a continuación:

Agraulis vanillae maculosa (Nymphalidae, Lepidoptera). Solía aparecer de a pares, aparentemente en cortejo. Realizaba un sobrevuelo circular sobre la inflorescencia, de hasta 20 segundos, luego se posaba sobre la inflorescencia, introducía la espiritrompa en una flor y succionaba, abriendo y cerrando las alas, entre 6 y 10 segundos. Dryas iulia alcionea (Nymphalidae, Lepidoptera). De mayor tamaño que los demás lepidópteros citados, volaba rápidamente. Inspeccionaba, posada con alas cerradas sobre la inflorescencia, y más frecuentemente libaba, con las alas extendidas, entre 2 y 4 segundos.

Ortilia ithra (Nymphalidae, Lepidoptera). Libaba sólo flores en fase temprana, posándose en las flores adyacentes a la flor libada.

Cymaenes odilia (Hesperiidae, Lepidoptera). Ahuyentaba a otros lepidópteros impidiendo que liben simultáneamente.
Epargyreus tmolis (Hesperiidae, Lepidoptera). Inspeccionaba posándose sobre la inflorescencia, sin evertir su espiritrompa. Luego de libar, solía permanecer posada en las hojas.

Apis mellifera (Apidae, Hymenoptera). Inspeccionaba sobrevolando o caminando sobre la inflorescencia. Al libar no introducía su cabeza en la corola.

Aff. Andreniidae y Halictidae (Hymenoptera). Se posaban sobre la corola y, a veces, intentaban introducir su cabeza dentro de la misma.

Bombus sp. y Xylocopa sp. (Apidae, Hymenoptera). Inspeccionaban caminando sobre la inflorescencia. Perforaban la corola y el cáliz.

Polystes sp. (Vespidae, Hymenoptera). Sobrevolaban lentamente las inflorescencias (aproximadamente 4 por mata). Ocasionalmente intentaban libar colgadas de las flores.

Brachygastra lecheguana (Vespidae, Hymenoptera). Sobrevolaban lentamente las inflorescencias describiendo círculos hasta posarse sobre las flores.

Syrphidae (Diptera). Se aproximaban lentamente a la flor y solían retroceder y volver a aproximarse inspeccionando cada flor de la inflorescencia. 


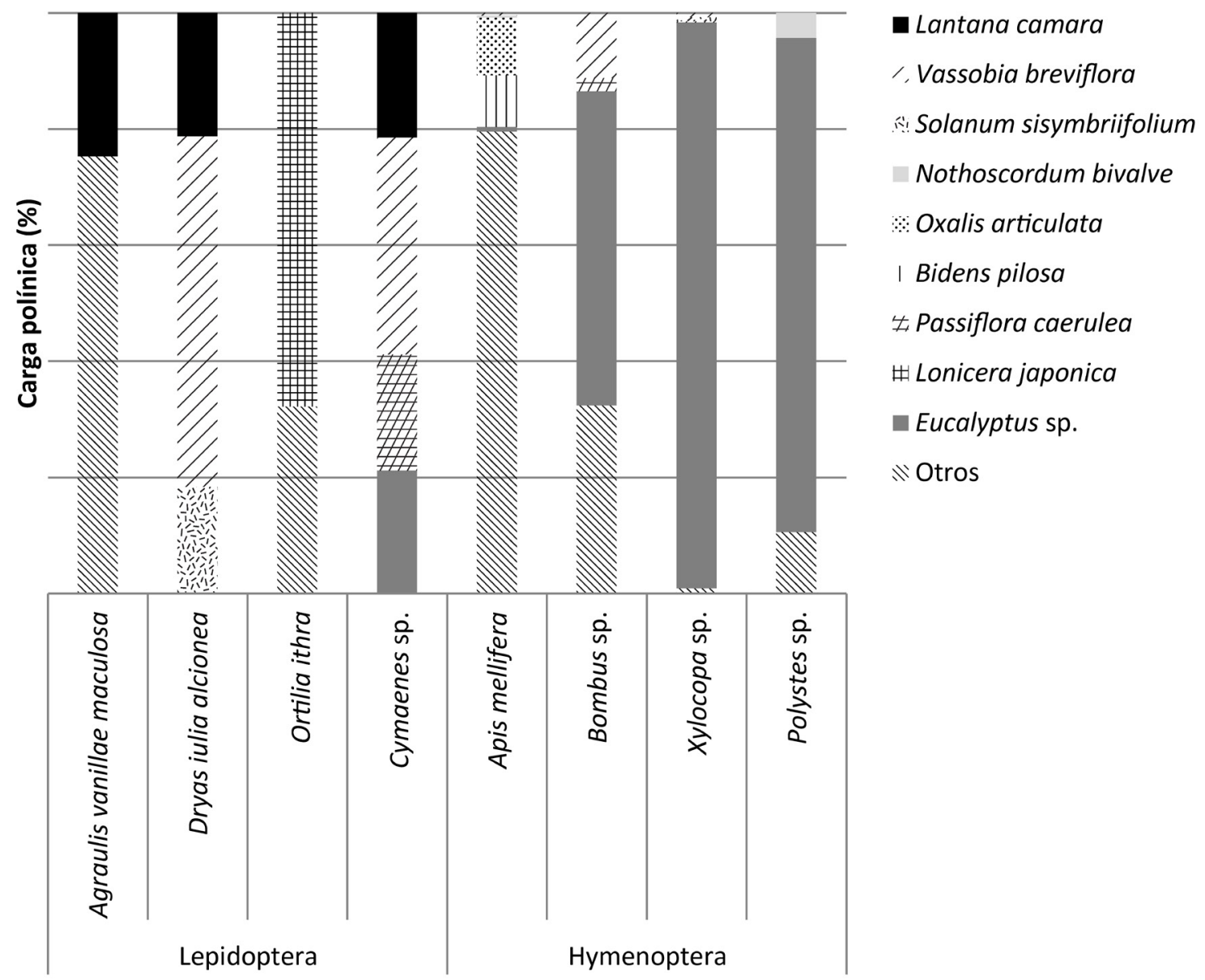

Fig. 4. Carga polínica de los insectos que visitaron las flores de $L$. camara y que transportaban polen.

Chlorostilbon lucidus (Trochilidae, Apodiformes). Visitaron sólo flores en fase temprana. Cada individuo libaba alrededor de 45 inflorescencias por mata en aproximadamente 10 minutos. Su actividad fue preponderante al atardecer. Libaron principalmente las inflorescencias situadas más arriba en los ejemplares de mayor porte.

\section{Discusión}

Sistema reproductivo. Los resultados obtenidos del experimento de polinización controlada son consistentes con los valores obtenidos para los diferentes índices calculados. En coincidencia con Neal (1999), se observó que puede ocurrir autopolinización espontánea, aunque el éxito reproductivo es significativamente mayor cuando actúan los polinizadores, constituyendo un sistema reproductivo predominantemente xenógamo. La hercogamia observada durante este ensayo sustenta este sistema. El valor obtenido del ISI indica que es parcialmente autoincompatible. Granja Barros et al. (2001) aseveraron que L. camara es autoincompatible: su trabajo se focaliza en variedades ornamentales, que pueden diferir en sus mecanismos genéticos de las poblaciones naturales. Según el índice P/O estimado, la especie sería xenógama facultativa, lo cual podría estar relacionado con su condición de invasora.

Antesis, fases florales y atractivos. La secuencia de la antesis de L. camara observada coincidió con la descripta por Barrows (1976), aunque la apertura de los capullos comenzó más tarde que 


\section{Bol. Soc. Argent. Bot. 54 (1) 2019}

lo informado por Granja Barros et al. (2001) y ocurrió en menos tiempo. Esto probablemente tenga relación con las diferentes condiciones de insolación y la influencia de ésta en la actividad de los polinizadores, ya que la polinización es lo que provoca el cambio de color, como constataron Mathur \& Ram (1978) mediante polinizaciones manuales. En el presente estudio el día 18, en que las flores alcanzaron la fase 2 más tarde y no pasaron a la fase 3, estuvo nublado y la frecuencia de visitas fue mucho menor, en contraste con los días 24 y 25. La fase temprana presentó el mayor volumen de néctar y la mayor duración, en coincidencia con los resultados obtenidos por Mathur \& Mohan Ram (1978) y Granja Barros et al. (2001), así como el único estadio de receptividad estigmática, reportado también por Mathur \& Mohan Ram (1978). El porcentaje de azúcares totales coincide con los valores registrados por Carrión-Tacuri et al. (2012), así como menor cantidad y ausencia de néctar en aproximadamente $65 \%$ de las flores naranjas y rojas, respectivamente (Carrión-Tacuri et al., 2012). El polen conservó su viabilidad a lo largo de la antesis.

La polinización desencadena una rápida síntesis de antocianinas, las cuales enmascaran a los carotenoides presentes en la corola, produciendo los cambios de coloración descriptos (Mohan Ram \& Mathur, 1984). El cambio de color de la corola orienta a los polinizadores hacia flores no polinizadas (Weiss, 1995). En el espectro $\mathrm{UV}$, el contraste entre el interior del tubo floral y el limbo constituiría guías de néctar visuales, indicando a los polinizadores el acceso a la recompensa, acumulada en la base de la corola, muy probablemente secretado por los tricomas que allí se hallaron. La presencia de pelos nectaríferos en la base de la corola en especies de Verbenaceae es común (Bernardello, 2007). La tinción con rojo neutro reveló la presencia de guías de néctar olfativas alrededor de la entrada al tubo floral, principalmente activas durante la fase temprana. Anderson \& Dobson (2003) observaron que los componentes exclusivos de la fragancia floral son vitales en el reconocimiento de recompensas alimenticias en flores de $L$. camara para mariposas de Heliconius melpomene (identificada como uno de los polinizadores) que realizan su primera visita a la planta, e interpretaron la coloración amarilla como el atractivo secundario que domina el comportamiento de estos polinizadores luego de haber sido recompensados con anterioridad; en consecuencia, deben reconocer que la corola roja no presenta recompensa, es decir, mientras el color sería el atractivo a corta distancia, el olor sería el atractivo secundario a larga distancia. El tipo estructural trompeta y el síndrome psicófilo ya reportado (Faegri \& Van Der Pijl, 1979) se completa con las guías nectaríferas olfativas y visuales encontradas en el presente trabajo.

El largo del tubo evitaría que los insectos de probóscide muy corta puedan acceder a la recompensa (Armstrong, 1979), por lo tanto la especie sería polinizada por insectos de probóscide suficientemente larga que acuden en busca de néctar. Los lepidópteros identificados poseen hábitos diurnos (Muthoka \& Mananze, 2005), en coincidencia con la antesis presentada por las flores. Poseen la visión bien desarrollada y pueden ver, a diferencia de otros insectos, el rojo y el amarillo, colores descriptos en las inflorescencias de $L$. camara. En general, son atraídos por olores dulces y frescos (Faegri \& Van Der Pijl, 1979). Liban posados; en este sentido el limbo desarrollado, en conjunto con el de otras flores de la inflorescencia, funciona como atractivo secundario y como plataforma de aterrizaje. Además, la persistencia de las flores que están finalizando la antesis sigue contribuyendo a la superficie de aterrizaje (Faegri \& Van Der Pijl, 1970; Weiss, 1991). En concordancia con los reportes previos, numerosos visitantes florales utilizaron las flores adyacentes a la flor a la cual iban a buscar recompensa como superficie de aterrizaje.

Visitantes florales. De los visitantes observados, Agraulis vanillae maculosa, Dryas iullia alcionea y Cymaenes sp. resultaron los polinizadores principales, siendo el primero el que mayor carga polínica de L. camara transportó y el segundo el que mayor número de visitas realizó. A su vez, el tiempo de forrajeo por inflorescencia, breve en ambos casos (menor en Dryas iullia alcionea) podría favorecer la xenogamia. En relación al polen transportado, se observó abundante presencia de granos de otros taxones en Agraulis vanillae maculosa, sugiriendo que esta especie sobrevuela áreas más extensas o tiene preferencias más generalistas. En los tres casos, la fase floral más visitada fue la temprana, y el mayor número de visitas se produjo por la tarde, si bien la actividad matutina 


\section{T. Amela García et al. - Biología reproductiva de Lantana camara}

de Cymaenes sp. fue también frecuente. La visita de Agraulis vanillae maculosa, aparentemente en situación de cortejo, podría tener relación con la descripción del síndrome de psicofilia realizado por Faegri \& Van Der Pijl (1979), quienes señalan la presencia de aminoácidos en el néctar de las especies psicófilas como componente esencial de la dieta de los lepidópteros asociada al cortejo. Agraulis vanillae maculosa fue mencionada como visitante floral de L. camara en otros sitios (Barrows, 1976; Schemske, 1976; Weiss, 1991; Granja Barros et al., 2001), pero sólo estos últimos autores corroboraron la transferencia polínica. Ortilia ithra forrajeó en las flores amarillas y la actividad se concentró principalmente por la tarde. Epargyreus tmolis se observó mayoritariamente en actividades de inspección y alimentación, mayoritariamente en la fase temprana. Como ninguna de estas especies transportaban polen de L. camara, serían hurtadores de néctar. Tampoco se encontró polen de L. camara sobre Apis mellifera; por otra parte, el comportamiento más frecuente consistió en la inspección y no la libación; por lo tanto, sería un hurtador de néctar cuando liba. Goulson \& Derwent (2004) interpretaron que esta abeja es el principal polinizador de L. camara en Australia, pero su suposición se basó en registro de visitas en relación a la producción de frutos, sin comprobar la transferencia de polen; en el caso que transfiera polen, lo haría vía la probóscide, al estilo de un lepidóptero, ya que, debido a sus dimensiones, es la única parte del cuerpo que podría acceder en forma legítima al interior del tubo floral. Los andrénidos y halíctidos registrados aparentemente libaban, principalmente flores amarillas, posándose sobre éstas; debido a su falta de transporte de polen, serían hurtadores de néctar. Los abejorros Bombus sp. y Xylocopa sp. no llevaban polen de L. camara, y por los cortes hallados en la corola de las flores recientemente visitadas por dichas especies, son ladrones de néctar. Acorde con estos resultados, Barrows (1976) enumera varios géneros de Apidae (entre éstos, Bombus y Xylocopa) como ladrones de néctar de varios géneros de plantas con corola tubular, los cuales perforan la corola por debajo de la base de los estambres, accediendo así al néctar. Respecto a los véspidos, tanto Brachygastra lecheguana, que libó mayoritariamente flores amarillas, como Polystes sp., que visitó flores en las tres fases (más asiduamente la intermedia), principalmente inspeccionando, pero no acarrearon polen de $L$. camara, serían hurtadores de néctar. Dado que los sírfidos principalmente inspeccionaron y no acarrearon polen durante el presente estudio, no se pueden considerar polinizadores pero tampoco se pueden sacar conclusiones respecto de otra función. Chlorostilbon lucidus no se pudo capturar de modo de comprobar si transportaba polen de L. camara, pero probablemente lo haga en su lengua, única parte que puede contactar los ciclos reproductivos, ocultos en la corola, al introducirla en ésta.

\section{Conclusiones}

Las características de psicofilia están bien representadas por las flores de L. camara, que, agrupadas en inflorescencias densas y virando del amarillo al rojo, pasando por el naranja, y exhibiendo guías de néctar simples en los espectros UV y visible, son polinizadas mayoritariamente por mariposas, que transportan el polen en la probóscide o regiones próximas a ésta. La poca carga polínica transportada por esta clase de vectores estaría compensada por las numerosas visitas de los polinizadores y por la capacidad de autopolinización espontánea.

Aunque el índice de autocompatibilidad indicó autocompatibilidad parcial y el índice $\mathrm{P} / \mathrm{O}$ predijo xenogamia facultativa, el mayor éxito reproductivo se alcanzó con polinización libre. El experimento reproductivo demostró que $L$. camara presenta capacidad de autopolinizarse espontáneamente, siendo ésta, probablemente, una de las causas de su propagación global.

\section{Contribución DE LOS AUtORES}

MTAG y MC diseñaron la investigación. DA, AB, MG, AHC, LDMA, MBM y VS realizaron conjuntamente y a partes iguales la colecta, análisis e interpretación de datos. MG redactó la primera versión del manuscrito. MTAG corrigió las versiones posteriores. DA realizó los análisis estadísticos. AB confeccionó las tablas. MG, MBM LDMA prepararon las figuras. VS compiló la bibliografía. AHC editó la versión final. 


\section{Agradecimientos}

Este manuscrito es producto del último trabajo guiado por la Dra. Patricia S. Hoc en su curso "Elementos de Biología Floral" en el Dpto. de Biodiversidad y Biología Experimental de la Facultad de Ciencias Exactas y Naturales de la Universidad de Buenos Aires. Desde los primeros hasta los últimos discípulos, estamos profundamente agradecidos por el entusiasmo con que nos hizo conocer esta fascinante disciplina.

\section{BiBLIOGRAFÍA}

AMELA GARCÍA, M. T. 1999. Biología floral y sistema reproductivo de especies nativas de Passiflora (Passifloraceae) de la Argentina. Tesis doctoral. Facultad de Ciencias Exactas y Naturales. Universidad de Buenos Aires. Buenos Aires, Argentina.

ANDERSSON, S. \& H. E. M. DOBSSON. 2003. Behavioral foraging responses by the butterfly Heliconius melpomene to L. camara floral scent. J. Chem. Ecol. 29: 2303-2318. https://doi.org/10.1023/A:1026226514968

ANTON, A.M. \& F. O. ZULOAGA. 2012. Verbenaceae. En: ESTUDIO SIGMA (eds.), Flora Argentina Vol. 14, pp. 1-220. Buenos Aires, Argentina.

ARMSTRONG, J. A. 1979. Biotic pollination mechanisms in the Australian flora - a review. New Zea. J. Bot. 17: 467-508. https://doi.org/10.1080/0028825X.1979.10432565

BAARS, J. R. \& S. NESER. 1999. Past and present initiatives on the biological control of $L$. camara (Verbenaceae) in South Africa. Afr. Entomol. 1: 21-33.

BARROWS, E. M. 1976. Nectar robbing and pollination of L. camara (Verbenaceae). Biotropica 8: 132-135. https://doi.org/10.2307/2989633

BEGUM, S., A. WAHAB; B. S. SIDDIQUI \& F. QAMAR. 2000. Nematicidal constituents of the aerial parts of $L$. camara. J. Nat. Prod. 63: 765-767. https://doi.org/10.1021/np9903548

BERNARDELLO, G. A. 2007. Systematic survey of floral nectaries. In: NICOLSON, S. W., M. NEPI. \& E. PACINI (eds.), Nectaries and nectar, pp. 19-128. Springer, London.

BHAGWAT, S. A., E. BREMAN, T. THEKAEKARA, T. F. THORNTON \& K. J. WILLIS. 2012. A battle lost? Report on two centuries of invasion and management of Lantana camara L. in Australia, India and South Africa. PloS one, 7(3), e32407. https://doi.org/10.1371/journal.pone.0032407

BRITO, M. F., C. H. TOKARINIA \& I. DÖBEREINER. 2004. A toxidez de diversas lantanas para bovinos e ovinos no Brasil. Pesqui. Vet. Bras. 24: 45-51. https://doi.org/10.1590/S0100-736X2004000300007
CAROPRESE ARAQUE, J. F., M. I. PARRA GARCÉS, D. ARRIETA PRIETO \& E. STASHENKO. 2011. Anatomía microscópica y metabolitos secundarios volátiles en tres estadios del desarrollo de las inflorescencias de L. camara (Verbenaceae). Rev. Biol. Trop. 59: 473-486.

CARRERE, R. 2006. El camará (L. camara): aportes para un mayor conocimiento sobre este arbusto indígena. Grupo Guayubira, Montevideo.

CARRIÓN-TACURI, J., R. BERJANO, G. GUERRERO, M. E. FIGUEROA, A. TYE \& J. M. CASTILLO. 2012. Nectar Production by Invasive L. camara and Endemic L. peduncularis in the Galápagos Islands. Pac. Sci. 66: 1-23. https://doi. org $/ 10.2984 / 66.4 .2$

CHAVAN, S. R. \& S. T. NIKAM. 1982. Investigation of Lantana camara Linn (Verbenaceae) leaves for larvicidal activity. B. Haffkine I. 10: 21-22.

CRUDEN, R. W. 1977. Pollen-ovule ratios: a conservative indicator of breeding systems in flowering plants. Evolution 31: 32-46. https://doi.org/10.2307/2407542

DAFNI, A. 1992. Pollination ecology. A practical approach. Princeton University Press, Princeton.

DENTON, G. R. W., R. MUNIAPPAN \& M. MARUTANI. 1991. The distribution and biological control of L. camara in Micronesia. Micronesica 3: 71-82.

DE MARZI, V. 2006. Cien plantas argentinas. Albatros, Buenos Aires.

ERDTMAN, G. 1952. Pollen morphology in plant taxonomy: Angiosperms. In: Almqvist \& Wiksell, Stockholm (eds.). 553 pp. Brill, Leiden.

FAEGRI, K. \& L. VAN DER PIJL. 1979. The Principles of Pollination Ecology. Pergamon Press, Oxford.

FENSHAM, R. J.; R. J. FAIRFAX, \& R. J. CANNELL. 1994. The invasion of L. camara L. in Forty Mile Scrub National Park, North Queensland. Aust. J. Ecol. 19: 297-305. https://doi.org/10.1111/j.1442-9993.1994.tb00493.x

GENISE, J., R. A. PALACIOS, P. S. HOC, R. CARRIZO, L. MOFFAT, M. P. MOM, M. A. AGULLÓ, P. PICCA \& S. TORREGOSA. 1990. Observaciones sobre la biología floral de Prosopis (Leguminosae, Mimosoideae). II. Fases florales y visitantes en el distrito chaqueño serrano. Darwiniana 30: 71-85.

GHISALBERTI, E. 2000. Review of L. camara L. Verbenaceae. Fitoterapia 71: 467- 486. https://doi.org/10.1016/S0367-326X(00)00202-1

GONCALVES, E., I. HERRERA, M. DUARTE, R. O. BUSTAMANTE, M. LAMPO, G. VELASQUEZ \& S. GARCÍA-RANGEL. 2014. Global invasion of Lantana camara: has the climatic niche been conserved across continents? PLoS One 9: e111468. https://doi.org/10.1371/journal.pone.0111468 


\section{T. Amela García et al. - Biología reproductiva de Lantana camara}

GOULSON, D. \& L. C. DERWENT. 2004. Synergistic interactions between an exotic honeybee and an exotic weed: pollination of L. camara in Australia. Weed Res. 44: 195-202. https://doi.org/10.1111/j.1365-3180.2004.00391.x

GRANJA BARROS, M.; V. RICO-GRAY \& C. DÍAZCASTELAZO. 2001. Sincronia de floracão entre $L$. camara (Verbenaceae) e Psittacanthus calyculatus (DC.) G. DON (Loranthaceae) ocorrentes nas dunas de la mancha, Veracruz, México. Acta Bot. Mex. 57: 1-14. https://doi.org/10.21829/abm57.2001.880

GRILLI, G. \& L. GALETTO. 2009. Remoción de frutos de una especie invasora (Lantana camara L.) en el Bosque Chaqueño de Córdoba (Argentina). Ecología Austral 19: 149-156.

HARLEY, K. L. S. \& R. C. KASSULKE. 1974. The suitability of Phytobia lantanae Frick for the biological control of L. camara in Australia. J. Aust. Entomol. Soc. 13: $229-233$. https://doi.org/10.1111/j.1440-6055.1974.tb02177.x

HASELER, W. H. 1966. The status of insects introduced for the biological control of weeds in Queensland. Aust. J. Entomol. 5: 1-4. https://doi.org/10.1111/j.1440-6055.1966.tb00668.x

HERNÁNDEZ, T., CANALES, M., AVILA, J. G., DURAN, A., CABALLERO, J., DE VIVAR, A. R., \& R. LIRA. 2003. Ethnobotany and antibacterial activity of some plants used in traditional medicine of Zapotitlán de las Salinas, Puebla (México). J. Ethnopharmacol. 88: 181-188. https://doi.org/10.1016/S0378-8741(03)00213-7

HERNÁNDEZ, M. P., S. M. CIVITELLA \& V. G. ROSATO. 2010. Uso medicinal popular de plantas y líquenes de la Isla Paulino, Provincia de Buenos Aires, Argentina. B. Latinoam. Caribe Plant. Med. Aromat. 9: 258 - 269.

HERRERA, C. M. 1987. Components of pollinator "quality": comparative analysis of a diverse insect assemblage. Oikos 50: 79-90. https://doi.org/10.2307/3565403

HEYWOOD, V. H. 1985. Las plantas con flores. In: REVERTÉ (eds.), pp. 332. Barcelona, España.

HOLM, L. G., D. PLUCKNETT, J. PANCHO \& J. HERBERGER. 1977. The World's Worst Weeds. Distribution and Biology. East West Center. In: UNIVERSITY PRESS OF HAWAII (eds.) pp. 609. University Press of Hawaii, Honolulu.

INOUYE, D. W. 1980. The terminology of floral larceny. Ecology 61: 1251-1253. https://doi.org/10.2307/1936841

KELLY, K. L. 1965. Standard sample $N^{\circ} 2106$ Supp. To Nat. Bur. Standards Cir. 553. Inter-Society Color Council- National Bureau of Standards colorname charts illustrated with centroid colors. U. S. Govemment Printing Office, Washington DC.
LOWE S. J., M. BROWNE \& S. BOUDJELAS. 2000. 100 of the World's Worst Invasive Alien Species. IUCN/SSC Invasive Species Specialist Group (ISSG), Auckland.

MATIENZO, Y., B. RAMOS \& E. RIJO. 2003. Revisión bibliográfica sobre L. camara L. Una amenaza para la ganadería. Redalyc 7: 45-55.

MATHUR, G. \& H. Y. MOHAN RAM. 1978. Significance of petal color in thrips-pollinated L. camara. L. Ann. Bot. 42: 1473-1476. https://doi.org/10.1093/oxfordjournals.aob.a085597

MOHAN RAM, H. Y. \& G. MATHUR. 1984. Flower color changes in L. camara. J. Exp. Bot. 35: 1656-1662.

MORTON, J. F. 1994. Lantana, or red sage (L. camara L, [Verbenaceae]), notorious weed and popular garden flower - some cases of poisoning in Florida. Econ. Bot. 48: 259-270.

MUTHOKA, C. N. \& S. MANANZE. 2005. Aspects of the pollination biology of L. camara (Verbenaceae). In: Abstracts from TBA Tanzania project reports on pollination, pp. 9. Tanzania.

NEAL, J. 1999. Assessing the sterility of ornamental Lantana varieties: Are we exacerbating the weed problem? Honor Thesis. Department of Botany, University of Queensland.

NÚÑEZ BUSTOS, E. 2008. Las especies urbanas de Rhopalocera de la Reserva Ecológica Costanera Sur, Ciudad de Buenos Aires, Argentina (Lepidoptera: Hesperioidea y Papilionoidea). SHILAP Rev. Lepidopt. 36: 435-447.

OSBORN, M. M., P.G. KEVAN \& M. A. LANE. 1988. Pollination biology of Opuntia polycantha and Opuntia phaecantha (Cactaceae) in Southern Colorado. Plant Syst. Evol. 159: 85-94. https://doi.org/10.1007/BF00937427

PERCIVAL, M. S. 1965. Floral biology. Pergamon Press, London. https://doi.org/10.1016/B978-0-08-010609-0.50009-1

R DEVELOPMENT CORE TEAM. 2009. R: A language and environment for statistical computing. R Foundation for Statistical Computing, Vienna, Austria. ISBN 3-900051-07-0, Disponible en: http://www.R-project.org.

RAMÍREZ-HERNÁNDEZ, S. G.,A. PÉREZ-VÁZQUEZ, J. C. GARCÍA-ALBARADO, A. GÓMEZ-GONZÁLEZ \& M. D. L. C. VARGAS-MENDOZA. 2012. Criterios para la selección de especies herbáceas ornamentales para su uso en paisajismo. Chapingo 18: 71-79.

RIVERA AMITA, M. M., I. HECHEVARRÍA SOSA, C. CARBALLO GUERRA \& M. REYES ARIAS. 2004. Posibilidades de control de enfermedades a partir de productos naturales y controles biológicos en las plantas medicinales. Rev. Cubana Plant. Med. [online]. Disponible en: http:// scielo.sld.cu/scielo.php?script $=$ sci_arttext\&pid $=$ S1028-47962004000300007. 
RUIZ ZAPATA, T. \& M. T. K. ARROYO. 1978. Plant reproductive ecology of a secondary deciduous tropical forest in Venezuela. Biotropica 10: 221230. https://doi.org/10.2307/2387907

SASTRY, C. T. \& K. Y. KAVATHEKAR. 1990. Plants for reclamation of wastelands. In: Publications and information directorate (eds.). Csir, New Delhi.

SCHEMSKE, D. W. 1976. Pollinator specificity in Lantana camara and L. trifolia (Verbenaceae). Biotropica 8: 260-264.

SHACKLETON, R. T., A. B. WITT, W. AOOL, \& C. F. PRATT. 2017. Distribution of the invasive alien weed, Lantana camara, and its ecological and livelihood impacts in eastern Africa. African Journal of Range \& Forage Science 34: 1-11.

SHARMA, S. \& O. P. SHARMA. 1989. An improved procedure for isolation and purification of lantadene A, the bioactive pentacyclic triterpenoid from L. camara leaves. JMAPS 21: 686-688.

SINGH, B., S. D. KHANDUJA \& G. S. SRIVASTAVA. 1984. Qualitative analysis of some firewood shrubs. Biomass 5: 317-320. https://doi.org/10.1016/0144-4565(84)90076-3

SUNDARAM, B. \& A. J. HIREMATH. 2012. Lantana camara invasion in a heterogeneous landscape: patterns of spread and correlation with changes in native vegetation. Biological Invasions 14: 1127-1141. https://doi.org/10.1007/s10530-011-0144-2
THAMAN, R. R. 1974. L. camara: its introduction, dispersal and impact on islands of the tropical Pacific Ocean. Micronesica 10: 17-39.

THORP, J. R. \& R. LYNCH. 2000. The determination of weeds of national significance. National weeds strategy executive committee, Launceston.

TOKARINIA, C. H. 1999. Estudos complementares sobre a toxidez de L. camara (Verbenaceae) em bovinos. Pesqui. Vet. Brasil. 19: 128-132. https://doi.org/10.1590/S0100-736X1999000300007

TRONCOSO, N. S. 1979. Verbenaceae. In: BURKART, A. (ed.), Flora de Entre Ríos, pp. 229-294. INTA, Buenos Aires.

VALLA, J. J. 1979. Morfología de las plantas superiores. Ed. Hemisferio Sur, Buenos Aires.

WELLS, M. J. \& C. H. STIRTON. 1988. Lantana camara: A poisonous declared weed. Farming in South Africa. Weeds A-27. Department of Agriculture and Water Supply, Pretoria.

WEISS, M. R. 1991. Floral colour changes as cues for pollinators. Nature 354: 227-229. https://doi.org/10.1038/354227a0

WEISS, M. R. 1995. Floral color change: A widespread functional convergence. Am. J. Bot. 82: $167-185$. https://doi.org/10.1002/j.1537-2197.1995.tb11486.x

WIKLUND, C., T. ERIKSSON \& H. LUNDBERG. 1979. The wood white butterfly Leptidea sinapis and its nectar plants: a case of mutualism or parasitism. Oikos 33: 358-362. https://doi.org/10.2307/3544323 